\title{
Contribution of anosmia and dysgeusia for diagnostic of COVID-19 in outpatients
}

\author{
Souheil Zayet ${ }^{1}$ - Timothee Klopfenstein ${ }^{1}$. Julien Mercier ${ }^{1} \cdot$ N'dri Juliette Kadiane-Oussou $^{1}$. \\ Ludovic Lan Cheong Wah ${ }^{1}$ - Pierre-Yves Royer ${ }^{1} \cdot$ Lynda Toko $^{1}$ - Vincent Gendrin ${ }^{1}$
}

Received: 21 April 2020 / Accepted: 7 May 2020 / Published online: 14 May 2020

(c) Springer-Verlag GmbH Germany, part of Springer Nature 2020

\section{Introduction}

Since its introduction to the human population in December 2019, Coronavirus Disease 2019 (COVID-19) continues to spread worldwide. More than $80 \%$ of patients with COVID19 present with influenza-like illness (ILI) or mild pneumonia [1], most of these patients are not hospitalized. Real time reverse transcriptase-polymerase chain reaction (RT-PCR) is used to detect the severe acute respiratory syndrome coronavirus 2 (SARS-CoV-2) from respiratory samples [2]. We aim to compare the symptoms of patients with positive and negative SARS-CoV-2 RT-PCR results and to determine the sensitivity, specificity, positive predictive value (PPV) and negative predictive value (NPV) for each of these symptoms in regard to SARS-CoV-2 RT-PCR.

\section{Methods}

Less than 30 miles from our hospital (Nord Franche-Comté Hospital), a major French cluster of COVID-19 began on March 1st, 2020 in Mulhouse city. We conducted a retrospective study between March, 30th and April, 3rd 2020, we collected the data of all adult patients ( $\geq 18$ years) who presented for possible COVID-19 at our outpatient department. Pregnant women, children $(<18$ years), patients with dementia (unable to report functional symptoms) were excluded.

During the beginning of the outbreak, we prospectively collected the symptoms of each patient consulting for a suspicion of COVID-19 to help us to screen the clinical feature of COVID-19. According to our data [3] and data of the

Souheil Zayet

souhail.zayet@gmail.com

1 Department of Infectious Diseases, Nord Franche-Comte

Hospital, 90400 Trévenans, France medical literature [1], we designed a standardized questionnaire to specify the symptoms in patients consulting for COVID-19 suspicion. Between March, 30th and April, 3rd 2020 we retrospectively collected the following data from the medical files of patients: demographic characteristics (age, sex), interval between illness onset and consultation, functional symptoms (measured fever $>38^{\circ} \mathrm{C}$, myalgia and/ or arthralgia, headache, cough, dyspnea, dysgeusia, anosmia, rhinorrhea, nausea, vomiting, diarrhea, and abdominal pain), clinical signs (crackling sounds heard on pulmonary auscultation) and result of RT-PCR SARS-CoV-2 nasopharyngeal sample. We didn't collect the outcome of the patients who were hospitalized.

Diagnosis was confirmed by real-time SARS-CoV-2 RT-PCR on nasopharyngeal swab specimen. For SARSCoV-2 real-time RT-PCR, viral RNA was extracted using the NucleoSpin ${ }^{\circledR}$ RNA Virus kit (Macherey-Nagel) according to the manufacturers' instructions, and amplified by RTPCR protocols developed by the Charité (E gene) [2] and the Institut Pasteur (RdRp gene) [4] on LightCycler 480® (Roche). Quantified positive controls were kindly provided by the French National Reference Center for Respiratory Viruses, Institut Pasteur, Paris.

We defined two groups of patients: Group 1 (G1: patients infected by COVID-19 confirmed by a positive RT-PCR) and Group 2 (G2: patients with a negative RT-PCR result on nasopharyngeal sample). Concerning the statistical analysis, continuous variables were expressed as mean and standard deviation (SD) and compared with ANOVA test. Categorical variables were expressed as number (\%) and compared by Chi square test or Fisher's exact test between the two groups (patients with confirmed COVID-19 and patients with negative SARS-CoV2 RT-PCR results). A $p$ value $<0.05$ was considered significant. We used the SPSS v24.0 software ${ }^{\circledR}$ (IBM, Armonk, NY, USA). 


\section{Results}

During the study period, 217 samples (nasopharyngeal swabs) were collected in our consultation: 95 patients (44\%) had a positive SARS-CoV-2 RT-PCR confirming the infection by COVID-19 and 122 patients (56\%) had a negative SARS-CoV-2 RT-PCR.

\section{Clinical description of patients with COVID-19}

In G1 (with positive SARS-CoV-2 RT-PCR; $n=95$ ), the mean age of patients was $40( \pm 12)$ years and $79(83 \%)$ were female. More than two-thirds of patients $(72 \%)$ were 31 to 60 years-old. The interval between illness onset and the consultation/ sample was 5.3 days $( \pm 2.8)$.

The most common symptoms ( $\geq 70 \%$ of cases) were cough $(79 \%, n=75)$, headache $(78 \%, n=74)$, myalgia $(75 \%$, $n=71)$ and fever $(74 \%, n=70)$. Otorhinolaryngological symptoms were present in more than half of the patients in G1: dysgeusia $(65 \%, n=62)$, anosmia $(63 \%, n=60)$ and rhinorrhea (at $62 \%, n=59)$. Only two patients (2/95) patients described isolated dysgeusia and/or anosmia as clinical symptoms in G1.

Fifty-four patients (57\%) had at least one gastro-intestinal (GI) symptom. Twenty four percent of patients $(n=23)$ had crackling sounds heard on pulmonary auscultation.

\section{Comparison between the two groups (Table 1)}

In our study, only 2 symptoms were statistically more frequent in G1 (PCR-positive) than in G2 (PCR-negative): dysgeusia $(65 \%$ vs $16 \%, p<0.001)$ and anosmia $(63 \%$ vs $15 \%, p<0.001)$. There were no significant differences in demographic characteristics and others clinical features between the two groups.

Amongst patients complaining of dysgeusia and anosmia, eleven patients were in G2 (PCR-negative) and 52 patients were in G1 (PCR-positive). Eighty-two percent $(n=9 / 11)$ of these 11 patients in $\mathrm{G} 2$ versus $38 \%(n=20 / 52)$ of the 52 patients in $\mathrm{G} 1$ were sampled more than 5 days after the onset of symptoms $(p=0.017)$.

\section{Sensitivity, specificity, PPV, and NPV of the symptoms (Table 2)}

The sensitivity of cough, headache, myalgia and fever was respectively $79 \%, 78 \%, 75 \%$ and $74 \%$ for a positive result of RT-PCR. However, the specificity of these symptoms was low. The specificity of anosmia, dysgeusia and crackling sounds heard on pulmonary auscultation was respectively of
$85 \%, 84 \%$ and $80 \%$. Specificity of the combination of anosmia and dysgeusia reached $91 \%$ for a positive PCR result. Dysgeusia and anosmia both had a positive predictive value of $77 \%$ for a positive RT-PCR result. The combination of these 2 symptoms had a positive predictive value of $83 \%$ for a positive SARS-CoV-2 RT-PCR result.

\section{Discussion}

This study describes a population of 217 outpatients presenting to our consultation for symptoms suggestive of COVID-19. Ninety-five patients (44\%) of these symptomatic adults were confirmed to be infected by COVID-19 by SARS-CoV-2 RT-PCR on nasopharyngeal sample, (in an area of intense SARS CoV-2 viral circulation). Most of these patients were health care workers (data not shown). This can explain the age distribution and the sex-ratio in this series.

In our study, the most common symptoms in the confirmed COVID-19 group were fever, cough, headache, myalgia and/or arthralgia as already described in medical literature. In a systematic review and meta-analysis (including 46,248 patients infected with COVID-19) by Yang et al. [5], the most common symptoms were fever, followed by cough, fatigue and dyspnea. In our population, dyspnea was reported by only $42 \%$ of patients (probably because they were outpatients, thus without any respiratory distress). We noticed two otorhinolaryngological symptoms: dysgeusia and anosmia, reported by more than half of the confirmed COVID-19 patients. These symptoms have been recently described related to SARS-CoV-2 [3, 6, 7]. Lechien et al. reported gustatory disorders in $89 \%$ of their patients $(n=342 / 417)$ and olfactory disorders in 86\% $(n=357 / 417)$ [6], these higher percentages may be explained by a selection bias with outpatients consulted at Otorhinolaryngology department.

In a recent publication, we showed that anosmia began $4.4( \pm 1.9$ [1-8]) days after infection onset [7]. The mean duration of anosmia was $8.9( \pm 6.3[1-21])$ days and $98 \%$ of patients recovered within 28 days [7].

In another study (not yet published), we reconstituted the history of onset of clinical symptoms of 70 patients with COVID-19: anosmia appears on average 5 days after the onset of the first other symptoms: pain syndrome appears first (on average 1.6 days after), then, fever in the second day (on average after 1.9 days) followed by cough (on average after 3.7 days) and diarrhea (on average after 4.5 days).

Patients with COVID-19 may also develop GI symptoms [8]. In the review of Li et al. [9], the over-all incidence of diarrhea was 5.8\% ( $n=145 / 2506)$. However in our study, GI symptoms were present in more than half of our patients, 
Table 1 Demographic characteristics and clinical data in patients with positive and negative SARS-CoV-2 RT-PCR on nasopharyngeal swab $(n=217)$

\begin{tabular}{|c|c|c|c|}
\hline Characteristics & $\begin{array}{l}\text { Positive RT-PCR } \\
\text { (COVID-19) } \\
(n=95)\end{array}$ & $\begin{array}{l}\text { Negative RT-PCR } \\
(n=122)\end{array}$ & $p$ value \\
\hline \multicolumn{4}{|l|}{ Demographic characteristics } \\
\hline Age (years) (mean, extremes, SD) & $\begin{array}{l}39.8[18-73] \\
\pm 12.2\end{array}$ & $\begin{array}{l}39.6[18-61] \\
\pm 11.7\end{array}$ & 0.889 \\
\hline \multicolumn{4}{|l|}{ Age range (number, \%) } \\
\hline [18-30] & $25(26.3)$ & $33(27)$ & 1 \\
\hline [31-40] & $24(25.3)$ & $30(24.6)$ & 1 \\
\hline$[41-50]$ & $26(27.4)$ & $27(22.1)$ & 0.427 \\
\hline$[51-60]$ & $18(18.9)$ & $27(22.1)$ & 0.615 \\
\hline$[61-70]$ & $1(1.1)$ & $2(1.6)$ & 1 \\
\hline$[71-80]$ & $1(1.1)$ & 0 & 0.438 \\
\hline \multicolumn{4}{|l|}{ Sex (number, \%) } \\
\hline Male & $16(16.8)$ & & 0.448 \\
\hline Female & $79(83.2)$ & $106(86.9)$ & 0.448 \\
\hline $\begin{array}{l}\text { Interval between illness onset and consultation } \\
\text { (days: mean, extremes, SD) }\end{array}$ & $\begin{array}{l}5.3[1-16] \\
\pm 2.8\end{array}$ & $\begin{array}{l}6.12[1-16] \\
\pm 3.3\end{array}$ & 0.118 \\
\hline \multicolumn{4}{|l|}{ Functional Signs } \\
\hline \multicolumn{4}{|l|}{ General symptoms } \\
\hline Fever $\geq 38{ }^{\circ} \mathrm{C}$ (number, $(\%)$ ) & $70(73.7)$ & $80(65.6)$ & 0.237 \\
\hline \multicolumn{4}{|l|}{ Pain symptoms } \\
\hline Myalgia and/or arthralgia (number, $(\%)$ ) & $71(74.7)$ & $79(64.8)$ & 0.221 \\
\hline Headache (number, $(\%)$ ) & $74(77.7)$ & $92(75.4)$ & 0.748 \\
\hline \multicolumn{4}{|l|}{ Respiratory symptoms } \\
\hline Cough (number, $(\%)$ ) & 75 (78.9) & $96(78.7)$ & 1 \\
\hline Dyspnea (number, $(\%)$ ) & $40(42.1)$ & $50(41)$ & 0.672 \\
\hline \multicolumn{4}{|l|}{ Otorhinolaryngological symptoms } \\
\hline Dysgeusia (number, $(\%)$ ) & $62(65.3)$ & $19(15.6)$ & $<0.001$ \\
\hline Anosmia $^{\mathrm{a}}$ (number, $(\%)$ ) & $60(63.2)$ & $18(14.8)$ & $<0.001$ \\
\hline Dysgeusia and anosmia (number, $(\%)$ ) & $52(54.7)$ & $11(9)$ & $<0.001$ \\
\hline Dysgeusia and/or anosmia (number, $(\%)$ ) & $70(73.7)$ & $27(22.1)$ & $<0.001$ \\
\hline Rhinorrhea (number, $(\%)$ ) & $59(62.1)$ & $77(63.1)$ & 0.524 \\
\hline Gastro-intestinal symptoms ${ }^{\mathrm{b}}$ (number, $(\%)$ ) & $54(56.8)$ & $69(56.6)$ & 0.965 \\
\hline \multicolumn{4}{|l|}{ Physical examination } \\
\hline $\begin{array}{l}\text { Crackling sounds heard on pulmonary auscul- } \\
\text { tation (number, }(\%) \text { ) }\end{array}$ & $23(24.2)$ & $23(18.9)$ & 0.099 \\
\hline
\end{tabular}

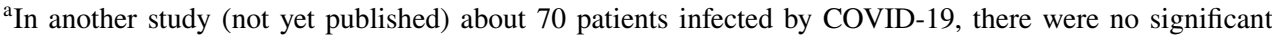
difference for viral load (VL) between patients with anosmia and patients without anosmia (5.45 [1.998.64] vs 5.27 [2.11 -8.51] log copies $/ \mathrm{ml}$ respectively, $p=0,67$ )

${ }^{b}$ Defined by: nausea and/or vomiting and/or diarrhea and /or abdominal pain which is similar to the incidence reported in the European study of Lechien et al. in outpatients [6].

In this epidemic context, the major finding of our work is the positive predictive value of anosmia (77\%), dysgeusia (77\%) and combination of anosmia plus dysgeusia (83\%) for a positive SARS-CoV-2 RT-PCR on nasopharyngeal sample. The main limitation for our study is that SARS-CoV-2 RTPCR on nasopharyngeal sample remains an insecure mean of diagnosis for patients with possible COVID-19. The current bibliography shows a sensitivity of 56-83\% for this test [10].
In our study, only nasopharyngeal SARS-CoV-2 RT-PCR was performed for diagnosis. RT-PCR for SARS-CoV-2 is very specific for COVID-19, but not enough sensitive. So, it is highly probable that some of the patients reporting anosmia and/or dysgeusia who tested negative for this RT-PCR may actually be infected by SARS-CoV-2 (false-negative results of the nasopharyngeal PCR test). Therefore, the PPV of these symptoms may even be higher for the diagnosis of COVID-19. The false negative rate of RT-PCR may be explained by a long interval between onset of the disease and PCR testing (as viral 
Table 2 Sensitivity, specificity, positive predictive value (PPV) and negative predictive value (NPV) of different symptoms for a positive result of SARS-CoV-2 RT-PCR on nasopharyngeal swab $(n=217)$

\begin{tabular}{|c|c|c|c|c|}
\hline Characteristics & Sensitivity (\%) & Specificity (\%) & PPV (\%) & NPV (\%) \\
\hline \multicolumn{5}{|l|}{ General symptoms } \\
\hline Fever $\geq 38{ }^{\circ} \mathrm{C}$ & 73.7 & 34.4 & 46.7 & 62.7 \\
\hline \multicolumn{5}{|l|}{ Pain symptoms } \\
\hline Myalgia and/or arthralgia & 74.7 & 34.7 & 47.3 & 63.6 \\
\hline Headache & 77.9 & 24.6 & 44.6 & 58.8 \\
\hline \multicolumn{5}{|l|}{ Respiratory symptoms } \\
\hline Cough & 78.9 & 21.3 & 43.9 & 56.5 \\
\hline Dyspnea & 42.1 & 58.7 & 44.4 & 56.3 \\
\hline \multicolumn{5}{|c|}{ Otorhinolaryngological symptoms } \\
\hline Dysgeusia & 65.3 & 84.4 & 76.5 & 75.7 \\
\hline Anosmia & 63.2 & 85.2 & 76.9 & 74.8 \\
\hline Dysgeusia and anosmia & 54.7 & 91 & 82.5 & 72.1 \\
\hline Dysgeusia and/or anosmia & 73.7 & 77.9 & 72.2 & 79.2 \\
\hline Rhinorrhea & 62.8 & 36.9 & 43.4 & 56.3 \\
\hline Gastro-intestinal symptoms ${ }^{\mathrm{a}}$ & 58.1 & 42.5 & 43.9 & 56.7 \\
\hline \multicolumn{5}{|l|}{ Physical examination } \\
\hline $\begin{array}{l}\text { Crackling sounds heard on } \\
\text { pulmonary auscultation }\end{array}$ & 24.5 & 79.8 & 50 & 56.2 \\
\hline
\end{tabular}

${ }^{a}$ Defined by: nausea and/or vomiting and/or diarrhea and/or abdominal pain load can decrease or become negative in nasopharyngeal samples during the second week of COVID-19) [11]. In our study, we noticed that $82 \%(n=9 / 11)$ patients, who complained of dysgeusia and anosmia with PCR-negative (G2), were sample more than 5 days after the onset of symptoms.

We emphasize the utility of prescribing a chest Computed Tomography (CT) scan to detect early changes due to COVID-19 in cases for which RT-PCR tests show negative results, despite a high clinical probability of COVID19 [12]. In our series, 23/112 patients in G2 had crackling sounds heard on pulmonary auscultation, and it is probable that some of them may have presented CT scan findings enabling us to validate a diagnosis of COVID-19. In recent studies, combination of RT-PCR (on respiratory samples) and serological tests also seems to enhance the sensitivity for COVID biological diagnosis [13].

Therefore, further studies are needed to find out the real PPV of anosmia and/or dysgeusia for COVID-19, using a multimodal diagnosing method $(\mathrm{RT}-\mathrm{PCR} \pm$ serology $\pm \mathrm{CT}$ scan). These positive predictive values for COVID-19 diagnosis will probably be even higher than the ones we found in our series with the sole nasopharyngeal SARS-CoV-2 RT-PCR.

\section{Conclusion}

Anosmia and/or dysgeusia are frequently reported by outpatients consulting for suspicion of COVID-19 (45\% of the patients in our series). It is important for clinician to know the predictive values of these symptoms. In this epidemic context, RT-PCR tests are not always widely available and sometimes with long delays. Outpatients presenting with dysgeusia and/or anosmia may be considered as patients infected with COVID-19, until microbiological confirmation has been obtained (as they have a high pre-test probability to be positive for SARS CoV-2 RT-PCR). Furthermore, for patients reporting dysgeusia and/or anosmia, with a negative RT-PCR SARS-CoV-2, it is necessary to remain vigilant before ruling out this diagnosis and to maintain hygiene precautions. It might be useful in these situations to get a deeper respiratory sample (sputum or naso-tracheal aspiration), and/ or to use other diagnostic tools (serology or thoracic CT scan).

Author contributions SZ, TK, JM, JNKO, LLCW collected the epidemiological and clinical data and processed statistical data. SZ and TK drafted the manuscript. LT, PYR and VG revised the final manuscript.

\section{Compliance with ethical standards}

Conflict of interest All authors declare no competing interests. We thank all patients involved in the study and especially Dr Zahra Hajer and Mrs Vogelbacher-Sattler Loane for their help.

\section{References}

1. Wu Z, McGoogan JM. Characteristics of and important lessons from the coronavirus disease 2019 (COVID-19) outbreak in China: summary of a report of 72,314 cases from the Chinese Center for Disease Control and Prevention. JAMA. 2020. 
2. Corman VM, Landt O, Kaiser M, Molenkamp R, Meijer A, Chu DKW, et al. Detection of 2019 novel coronavirus (2019-nCoV) by real-time RT-PCR. Euro Surveill. 2020;25:1.

3. Zayet S, N'dri Juliette K-O, Royer P-Y, Toko L, Gendrin V, Klopfenstein T. Coronavirus disease 2019: new things to know! J Med Virol. 2020.

4. Bernard Stoecklin S, Rolland P, Silue Y, Mailles A, Campese C, Simondon A, et al. First cases of coronavirus disease 2019 (COVID-19) in France: surveillance, investigations and control measures, January 2020. Euro Surveill. 2020;25:1.

5. Yang J, Zheng Y, Gou X, Pu K, Chen Z, Guo Q, et al. Prevalence of comorbidities in the novel Wuhan coronavirus (COVID-19) infection: a systematic review and meta-analysis. Int J Infect Dis. 2020 Mar 12;

6. Lechien JR, Chiesa-Estomba CM, De Siati DR, Horoi M, Le Bon $\mathrm{SD}$, Rodriguez A, et al. Olfactory and gustatory dysfunctions as a clinical presentation of mild-to-moderate forms of the coronavirus disease (COVID-19): a multicenter European study. Eur Arch Otorhinolaryngol. 2020.

7. Klopfenstein T, Kadiane-Oussou NJ, Toko L, Royer P-Y, Lepiller $\mathrm{Q}$, Gendrin V, et al. Features of anosmia in COVID-19. Med Mal Infect. 2020.
8. Xiao F, Tang M, Zheng X, Liu Y, Li X, Shan H. Evidence for gastrointestinal infection of SARS-CoV-2. Gastroenterology. 2020.

9. Li X-Y, Dai W-J, Wu S-N, Yang X-Z, Wang H-G. The occurrence of diarrhea in COVID-19 patients. Clin Res Hepatol Gastroenterol. 2020.

10. Kokkinakis I, Selby K, Favrat B, Genton B, Cornuz J. Covid-19 diagnosis : clinical recommendations and performance of nasopharyngeal swab-PCR. Rev Med Suisse. 2020;16:699-701.

11. To KK-W, Tsang OT-Y, Leung W-S, Tam AR, Wu T-C, Lung DC, et al. Temporal profiles of viral load in posterior oropharyngeal saliva samples and serum antibody responses during infection by SARS-CoV-2: an observational cohort study. Lancet Infect Dis. 2020.

12. Ai T, Yang Z, Hou H, Zhan C, Chen C, Lv W, et al. Correlation of chest CT and RT-PCR testing in coronavirus disease 2019 (COVID-19) in China: A report of 1014 cases. Radiology. 2020;26:200642.

13. Zhao J, Yuan Q, Wang H, Liu W, Liao X, Su Y, et al. Antibody responses to SARS-CoV-2 in patients of novel coronavirus disease 2019. Clin Infect Dis. 2020. https://doi. org/10.1101/2020.03.02.20030189. 